\title{
Pre-Analytical Factors Influence Vitamin D Measurement In Clinical Laboratory
}

\author{
Fahima A Alnagar \\ University of Tripoli, Faculty of Veterinary Medicine, Department of Physiology, Biochemistry and \\ Nutrition, Tripoli, Libya.
}

\begin{abstract}
:
It has been more than 90 years since the discovery of vitamin D and its capability to cure rickets in children. Recently, awareness is growing of the prevalence of vitamin D deficiency in the general population in association with an increased risk of several diseases. Analyses of vitamin D levels in blood samples provide direct measures of exposure to dietary or supplemented vitamin. This review is focused on the pre-analytical factors and challenges are known to affect the concentrations of vitamin D biomarkers. The pre-analytical measurement of disease relevant vitamin D deficiency in blood can vary as a function of differences in sample handling and processing. It could impact the accurate measurement of the diseases relevant vitamin D deficiency. Majority of the analysis in a clinical biochemistry laboratory requires serum or plasma as the analytical specimen and obtaining serum or plasma from a primary sample is an important step of pre-analytical procedure. Although vitamin D is considered as a relatively stable analyte, effect of pre-analytical conditions and stability of vitamin D in serum and plasma needs to be identified clearly.
\end{abstract}

Key Words: Vitamin D, Vitamin D binding protein (DBP), Pre-analytical factors, Vitamin D measurements

\section{Introduction:}

\subsection{Vitamin D metabolites measurements in the blood circulation}

Vitamin D is a generic name for a heterogeneous group of anti-rachitic substances [1]. It is an essential nutrient of the human diet. There are two biological precursors of vitamin $D$; the plant origin form is ergocalciferol $\left(D_{2}\right)$ and cholecalciferol $\left(D_{3}\right)$ which is synthesized in the human skin and can be consumed in the form of oily fish, fortified foods, or dietary supplements [1, 2]. The two forms differ in the structures of their side chains, but they are metabolized identically and have equivalent biological activities. Vitamin D is metabolized to several forms, the primary is converted to 25-hydroxyvitamin D $(25(\mathrm{OH}) \mathrm{D})$ in the liver which then converted by the enzyme $1 \alpha$-hydroxylase to the active form, 1,25-dihydroxyvitamin $\mathrm{D}(1,25(\mathrm{OH}) 2 \mathrm{D})$ [3]. However, 25-OH-D is the predominant circulating form of vitamin D which is considered to be the most reliable index of human's vitamin D status and currently used in clinical practice to asses it [4].

In the circulation, vitamin D like other steroid hormones is bound tightly to a special carrier vitamin D binding protein (DBP) and smaller amounts are bound to blood proteins, albumin and lipoproteins [5]. Only very tiny amounts of the total vitamin D are free and potentially biologically active. It was reported that $85 \%$ of vitamin D metabolites are circulated in the blood bounded to DBP, about $15 \%$ are loosely bound to serum albumin and only tiny amounts (less than $0.03 \%$ ) exist in free, unbounded form [6]. The fraction of "bioavailable" vitamin D metabolites consists of the fraction of the free vitamin D and the albumin-bound fraction which then measures around 15\% in normal individuals [7]. DBP has the higher binding affinity with 25OHD form than 1,25OHD because the later has an additional hydroxyl group which changes metabolite's molecular geometry [8]. The molar concentration of DBP in blood is more than 20-fold higher compared to the molar concentration of all vitamin D forms [9].

Vitamin D deficiency is pandemic, widespread and is re-emerging as a major health problem globally [10, 11]. A worldwide rapid and dramatic increase in 25OHD assays has been observed over the last years $[12,13]$. The assessment of vitamin D is critically important because it plays a major role in calcium and bone metabolism and is essential in cell maturation and proliferation [14]. Deficiency of vitamin D is related to an increased risk of rickets [15], cardiovascular disease [16], colon cancer [17, 18], uterine fibroids [19] and some other diseases such as diabetes type 2 [20], psoriasis[21] and thyroid disease [22], rheumatoid arthritis [23], Alzheimer's disease [24] and Parkinson's disease [25]. Undetected vitamin D deficiency may increase a patient's risk of these diseases. Therefore, the measurement of $25 \mathrm{OHD}$ is becoming increasingly important in the management of patients with various disorders and diseases. In addition, due to the widespread of vitamin D deficiency and its potential impact on health, awareness is growing of the need for accurate assessment of vitamin D status in the general population and epidemiological studies. Issues relating to the measurement of vitamin D biomarkers can be broadly divided into three categories; pre-analytical, analytical and post-analytical factors. All these factors may affect the measurement of vitamin D either separately or together. The pre-analytical factors were assessed in the aspects of clinical laboratory and epidemiological studies. This review will concentrate on the common pre-analytical factors may affect the measurement of vitamin D in clinical laboratories. 


\subsection{Pre-analytical stability of vitamin D}

Large scale epidemiological studies designed to evaluate the association between serum or plasma levels of an analyte and risk of disease. These studies frequently require long intervals of storage time between blood collection and laboratory analysis. Thus, the stability of samples under the relevant storage and processing conditions must be known prior to using such samples to test these analytes. Assaying vitamin D is relatively expensive and is therefore often lost in large clinical trials [26]. These assays are extremely variable which confounds the diagnosis of vitamin D insufficiency or deficiency and may produce inaccurate results [27]. This variation was related to the instability of $25 \mathrm{OHD}$ or $1,25 \mathrm{OHD}$ in blood or serum exposed to various factors during sample processing, shipping, and storage conditions. Optimization of these conditions is necessary to minimize these pre-analytical errors. Some studies have evaluated the effect of pre-analytical factors on $25 \mathrm{OHD}$ or 1,25OHD samples. factors including the influence of temperature, freeze-thaw cycles, centrifugation, tube types and light will be discussed.

\subsubsection{Influence of temperature on vitamin D metabolites}

There are few studies explored the effect of temperature on the stability of vitamin D metabolites either in whole blood, plasma or serum. The results of these studies were controversy due to some limitations such as small sample size and imprecision of the assay used. A study assessed the stability of different vitamin D metabolites including 1,25OHD and 24,25OHD reported that 1,25OH in serum is stable for 57 days at $-80{ }^{\circ} \mathrm{C}, 24 \mathrm{~h}$ at $4{ }^{\circ} \mathrm{C}$, and unstable at room temperature [28]. Similarly, older studies investigated the stability of vitamin D in samples stored at $-20{ }^{\circ} \mathrm{C}$ showed $10 \%$ decrease in vitamin D concentration after 3 months [29, 30]. On the other hand, a study evaluated a short term vitamin D stability in serum and plasma samples reported no significant change at 24 ${ }^{\circ} \mathrm{C}$ during 72 hours [31]. Moreover, another studies found that 25OHD and 1,25OHD are stable in serum at physiological concentrations at room temperature or under refrigeration for at least 2 weeks [32, 33]. These studies have related this finding to that $25 \mathrm{OHD}$ and $1,25 \mathrm{OH}$ are extremely stable compounds, as they are bound to vitamin D binding protein (DBP).

\subsubsection{Influence of centrifugation}

In order to obtain plasma or serum samples for the processing of vitamin D metabolites assays, centrifugation of the blood samples is needed. It is a part of the pre-analytical stage and can be done with or without temperature controlled centrifuges. A recent study which was the first investigated the effect of centrifugation temperature on vitamin $\mathrm{D}$ concentration reported that there were no differences between refrigerated centrifuge $\left(2-8{ }^{\circ} \mathrm{C}\right)$ and centrifuge at room temperature [34]. In addition, some have questioned whether delayed centrifuging reduces vitamin D stability due to hemolysis [35]. Previous studies illustrated that delaying centrifugation of blood samples prior to vitamin D metabolites analysis has no significant differences up to 96 hour after blood collection $[36,37]$. These results suggested that vitamin D requires no special care in terms of centrifugation.

\subsubsection{Influence of repeated freeze thaw cycles}

Prospective studies can store serum from participants which offer the opportunity to further study the relationship between vitamin $\mathrm{D}$ deficiency and related diseases. Analyzing large-scale freshly collected samples in one time is impractical and prohibitively expensive. Consequently, these large-scale studies require refreezing and thawing samples more than once which may adversely affect the measurements of the analyte. In this context, no adverse effect was found on the repeated freeze-thawing cycles of vitamin D concentration, although repeated freeze-thaw cycles should be avoided [38, 39].

\subsubsection{Influence of tube types used to collect blood samples}

Different types of tubes may be used to collect blood sample for analysis of vitamin D metabolites. The available choices of these tubes used are; two different plasma anticoagulant tubes containing either (heparin or EDTA), red top serum tubes or gel tubes. Concerns have been raised regarding using serum or plasma which may affect the concentration of vitamin D measurements [40]. Although there was a little information about the potential impact of blood collection tubes on vitamin D concentrations, conflicted results were reported by two different studies. The first found that sample type did not affect vitamin D stability and both serum and plasma could be used for storage [34], while the second study reported difference in vitamin D concentrations according to tube type in some assays for heparinized plasma [36]. In addition, gel tubes were also used for collecting blood centrifuged to produce serum. This type of tubes contains gel which forms a barrier after centrifugation and has markedly improved serum analyte stability in the primary tube and removed the need for aliquoting the serum [41]. A study by Tanner and colleagues assessed the effects of temperature and time on serum samples collected into gel tubes prior to centrifugation. Overall, most analytes including vitamin D were stable and provided valid results [37]. In contrast, another study found that gels have adverse effects for 25OHD [42]. Hence blood for $25 \mathrm{OH} \mathrm{D}$ measurement is probably best collected into plain tubes without anticoagulants or gels.

\subsubsection{Influence of light}

Although protection of specimens from light after blood collection in some studies was recommended for accurate vitamin D analysis [43, 44], the potential impact of light has not been extensively studied. Few reports examined the adverse effect of light on vitamin D metabolites [31, 39, 45]. Thes studies reported that vitamin D is unaffected by pre-analytical exposure of light experienced in medical laboratories. Moreover, a study evaluated the effect of ultraviolet light on crystalline and serum or plasma $25 \mathrm{OHD}$ or $1,25 \mathrm{OHD}$, reported that the UV light destroyed the crystalline $25 \mathrm{OHD}$ or 1,250HD within a few minutes but the serum or plasma 25OHD and 1,25OHD levels did not change after 2 days of UV light exposure. These studies suggested that the stability of vitamin D and its metabolites in serum or plasma when they are insulted with UV light is related to the high bounding affinity of vitamin D to the serum DBP which are resistant to the UV light [46]. 


\section{Conclusion}

In large epidemiological studies and large clinical samples, it is not feasible to centrifuge and freeze blood samples immediately after collection. This delay of processing samples may affect the quality of the analytes. The impact of pre-analytical variability on measured vitamin D values is necessary to minimize pre-analytical errors. Although the concentration of vitamin D was reported to be "solid as a rock" under the common pre-analytical conditions experienced in medical laboratories, the routine measurement of vitamin D faces several challenges attributable to the pre-analytical factors. These conditions need to be more investigated and standardized with larger sample size and consider the analytical and post-analytical factors.

\section{References}

[1] Norman AW. From vitamin D to hormone D: fundamentals of the vitamin D endocrine system essential for good health. The American journal of clinical nutrition. 2008;88(2):491s-9s.

[2] Lee JH, O'Keefe JH, Bell D, Hensrud DD, Holick MF. Vitamin D deficiency an important, common, and easily treatable cardiovascular risk factor? Journal of the American College of Cardiology. 2008;52(24):1949-56.

[3] DeLuca HF. Overview of general physiologic features and functions of vitamin D. The American journal of clinical nutrition. 2004;80(6 Suppl):1689s-96s.

[4] Preece MA, TomlinsonS, Ribot CA, Pietrek J, Korn HT, Davies DM, et al. Studies of vitamin D deficiency in man. The Quarterly journal of medicine. 1975;44(176):575-89.

[5] Cooke NE, Haddad JG. Vitamin D binding protein (Gc-globulin). Endocrine reviews. 1989;10(3):294-307.

[6] Bikle DD, Malmstroem S, Schwartz J. Current Controversies: Are Free Vitamin Metabolite Levels a More Accurate Assessment of Vitamin D Status than Total Levels? Endocrinology and metabolism clinics of North America. 2017;46(4):901-18.

[7] Mendel CM. Rates of dissociation of sex steroid hormones from human sex hormone-binding globulin: a reassessment. The Journal of steroid biochemistry and molecular biology. 1990;37(2):251-5.

[8] Mason D, Donabella PJ, Nnani D, Musteata FM. Normalized vitamin D metabolite concentrations are better correlated to pharmacological effects than measured concentrations. Future science OA. 2015;1(4):Fso83.

[9] Szabo B, Tabak AG, Toldy E, Szekeres L, Szili B, Bakos B, et al. The role of serum total and free 25hydroxyvitamin D and PTH values in defining vitamin D status at the end of winter: a representative survey. Journal of bone and mineral metabolism. 2017;35(1):83-90.

[10] van der Meer IM, Middelkoop BJ, Boeke AJ, Lips P. Prevalence of vitamin D deficiency among Turkish, Moroccan, Indian and sub-Sahara African populations in Europe and their countries of origin: an overview. Osteoporosis international : a journal established as result of cooperation between the European Foundation for Osteoporosis and the National Osteoporosis Foundation of the USA. 2011;22(4):1009-21.

[11] Mithal A, Wahl DA, Bonjour JP, Burckhardt P, Dawson-Hughes B, Eisman JA, et al. Global vitamin D status and determinants of hypovitaminosis D. Osteoporosis international : a journal established as result of cooperation between the European Foundation for Osteoporosis and the National Osteoporosis Foundation of the USA. 2009;20(11):1807-20.

[12] Bilinski K, Boyages S. The rise and rise of vitamin D testing. BMJ (Clinical research ed). 2012;345:e4743.

[13] Caillet P, Goyer-Joos A, Viprey M, Schott AM. Increase of vitamin D assays prescriptions and associated factors: a population-based cohort study. Scientific reports. 2017;7(1):10361.

[14] Holick MF. Vitamin D deficiency. The New England journal of medicine. 2007;357(3):266-81.

[15] Holick MF. Resurrection of vitamin D deficiency and rickets. The Journal of clinical investigation. 2006;116(8):2062-72.

[16] Wang TJ, Pencina MJ, Booth SL, Jacques PF, Ingelsson E, Lanier K, et al. Vitamin D deficiency and risk of cardiovascular disease. Circulation. 2008;117(4):503-11.

[17] Tangrea J, Helzlsouer K, Pietinen P, Taylor P, Hollis B, Virtamo J, et al. Serum levels of vitamin D metabolites and the subsequent risk of colon and rectal cancer in Finnish men. Cancer causes \& control : CCC. 1997;8(4):615-25.

[18] Klampfer L. Vitamin D and colon cancer. World journal of gastrointestinal oncology. 2014;6(11):430-7.

[19] Baird DD, Hill MC, Schectman JM, Hollis BW. Vitamin d and the risk of uterine fibroids. Epidemiology (Cambridge, Mass). 2013;24(3):447-53.

[20] Rafiq S, Jeppesen PB. Is Hypovitaminosis D Related to Incidence of Type 2 Diabetes and High Fasting Glucose Level in Healthy Subjects: A Systematic Review and Meta-Analysis of Observational Studies. Nutrients. 2018;10(1).

[21] Barrea L, Savanelli MC, Di Somma C, Napolitano M, Megna M, Colao A, et al. Vitamin D and its role in psoriasis: An overview of the dermatologist and nutritionist. Reviews in endocrine \& metabolic disorders. 2017;18(2):195-205. 
[22] Kim D. The Role of Vitamin D in Thyroid Diseases. International journal of molecular sciences. 2017;18(9).

[23] Kostoglou-Athanassiou I, Athanassiou P, Lyraki A, Raftakis I, Antoniadis C. Vitamin D and rheumatoid arthritis. Therapeutic advances in endocrinology and metabolism. 2012;3(6):181-7.

[24] Littlejohns TJ, Henley WE, Lang IA, Annweiler C, Beauchet O, Chaves PH, et al. Vitamin D and the risk of dementia and Alzheimer disease. Neurology. 2014;83(10):920-8.

[25] Ross GW, Petrovitch H, Abbott RD. Serum vitamin D and risk of Parkinson's disease. Movement disorders : official journal of the Movement Disorder Society. 2016;31(7):993-35.

[26] Bilinski K, Boyages S. Evidence of overtesting for vitamin D in Australia: an analysis of 4.5 years of Medicare Benefits Schedule (MBS) data. BMJ open. 2013;3(6).

[27] Wallace AM, Gibson S, de la Hunty A, Lamberg-Allardt C, Ashwell M. Measurement of 25-hydroxyvitamin D in the clinical laboratory: current procedures, performance characteristics and limitations. Steroids. 2010;75(7):477-88.

[28] Duan X, Weinstock-Guttman B, Wang H, Bang E, Li J, Ramanathan M, et al. Ultrasensitive quantification of serum vitamin $D$ metabolites using selective solid-phase extraction coupled to microflow liquid chromatography and isotope-dilution mass spectrometry. Analytical chemistry. 2010;82(6):2488-97.

[29] Norris RL, Thomas MJ, Craswell PW. Assessment of a two-step high-performance liquid chromatographic assay using dual-wavelength ultraviolet monitoring for 25-hydroxyergocalciferol and 25hydroxycholecalciferol in human serum or plasma. Journal of chromatography. 1986;381(1):53-61.

[30] Ocke MC, Schrijver J, Obermann-de Boer GL, Bloemberg BP, Haenen GR, Kromhout D. Stability of blood (pro)vitamins during four years of storage at -20 degrees $\mathrm{C}$ : consequences for epidemiologic research. Journal of clinical epidemiology. 1995;48(8):1077-85.

[31] Lissner D, Mason RS, Posen S. Stability of vitamin D metabolites in human blood serum and plasma. Clinical chemistry. 1981;27(5):773-4.

[32] Carter GD, Carter CR, Gunter E, Jones J, Jones G, Makin HL, et al. Measurement of Vitamin D metabolites: an international perspective on methodology and clinical interpretation. The Journal of steroid biochemistry and molecular biology. 2004;89-90(1-5):467-71.

[33] El-Khoury JM, Wang S. Stability of 1,25-dihydroxyvitamin $D(2)$ and 1,25-dihydroxyvitamin $D(3)$ in human serum. Clinical biochemistry. 2012;45(9):707-8.

[34] Colak A, Toprak B, Dogan N, Ustuner F. Effect of sample type, centrifugation and storage conditions on vitamin D concentration. Biochemia medica. 2013;23(3):321-5.

[35] Landi MT, Caporaso N. Sample collection, processing and storage. IARC scientific publications. 1997(142):223-36.

[36] Yu CL, Falk RT, Kimlin MG, Rajaraman P, Sigurdson AJ, Horst RL, et al. The impact of delayed blood centrifuging, choice of collection tube, and type of assay on 25-hydroxyvitamin D concentrations. Cancer causes \& control : CCC. 2010;21(4):643-8.

[37] Tanner M, Kent N, Smith B, Fletcher S, Lewer M. Stability of common biochemical analytes in serum gel tubes subjected to various storage temperatures and times pre-centrifugation. Annals of clinical biochemistry. 2008;45(Pt 4):375-9.

[38] Antoniucci DM, Black DM, Sellmeyer DE. Serum 25-hydroxyvitamin D is unaffected by multiple freeze-thaw cycles. Clinical chemistry. 2005;51(1):258-61.

[39] Wielders JP, Wijnberg FA. Preanalytical stability of 25(OH)-vitamin D3 in human blood or serum at room temperature: solid as a rock. Clinical chemistry. 2009;55(8):1584-5.

[40] Yin P, Lehmann R, Xu G. Effects of pre-analytical processes on blood samples used in metabolomics studies. Analytical and bioanalytical chemistry. 2015;407(17):4879-92.

[41] Boyanton BL, Jr., Blick KE. Stability studies of twenty-four analytes in human plasma and serum. Clinical chemistry. 2002;48(12):2242-7.

[42] Lensmeyer GL, Wiebe DA, Binkley N, Drezner MK. HPLC method for 25-hydroxyvitamin D measurement: comparison with contemporary assays. Clinical chemistry. 2006;52(6):1120-6.

[43] Sadat-Ali M, Al-Elq AH, Al-Shaikh IH, Al-Turki HA, Al-Ali AK, Al-Othman AA. Assessment of low vitamin D among Saudi Arabians. Did we overshoot the runway? Saudi medical journal. 2014;35(10):1243-9.

[44] Yousef FM, Jacobs ET, Kang PT, Hakim IA, Going S, Yousef JM, et al. Vitamin D status and breast cancer in Saudi Arabian women: case-control study. The American journal of clinical nutrition. 2013;98(1):105-10.

[45] Lewis JG, Elder PA. Serum 25-OH vitamin D2 and D3 are stable under exaggerated conditions. Clinical chemistry. 2008;54(11):1931-2.

[46] Hollis BW. Measuring 25-hydroxyvitamin D in a clinical environment: challenges and needs. The American journal of clinical nutrition. 2008;88(2):507s-10s. 
